\title{
THE INSPIRE RESEARCH INITIATIVE: A PROGRAM FOR GEROSCIENCE AND HEALTHY AGING RESEARCH GOING FROM ANIMAL MODELS TO HUMANS AND THE HEALTHCARE SYSTEM
}

\author{
P. DE SOUTO BARRETO ${ }^{1,2, *}$, S. GUYONNET ${ }^{1,2, *}$, I. ADER ${ }^{3}$, S. ANDRIEU $^{1,2}$, L. CASTEILLA $^{3}$, \\ N. DAVEZAC ${ }^{4}$, C. DRAY ${ }^{5}$, N. FAZILLEAU ${ }^{6}$, P. GOURDY $^{5}$, R. LIBLAU $^{6}$, A. PARINI $^{5}$, P. PAYOUX ${ }^{7}$,

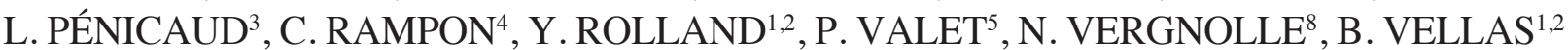 \\ FOR THE INSPIRE PROGRAM GROUP
}

\begin{abstract}
1. Gerontopole of Toulouse, Institute of Ageing, Toulouse University Hospital (CHU Toulouse), Toulouse, France; 2. UPS/Inserm UMR1027, University of Toulouse III, Toulouse, France; 3. STROMALab, Etablissement Français du Sang-Occitanie (EFS), Inserm 1031, University of Toulouse, National Veterinary School of Toulouse (ENVT), ERL5311 CNRS, Toulouse, France; 4. Centre de Recherches sur la Cognition Animale (CRCA), Centre de Biologie Intégrative (CBI), Université de Toulouse, CNRS, UPS, Toulouse, France ; 5. Institut des Maladies Métaboliques et Cardiovasculaires, Inserm/Université Paul Sabatier UMR 1048 - I2MC 1 avenue Jean Poulhès BP 8422531432 Toulouse Cedex 4 - France; 6. Centre de

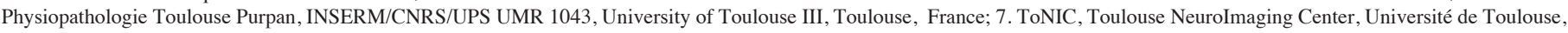
Inserm, UPS, France; 8. IRSD, Université de Toulouse, INSERM, INRA, ENVT, UPS, U1220, CHU Purpan, CS60039, 31024, Toulouse, France.

*These authors contributed equally to this work

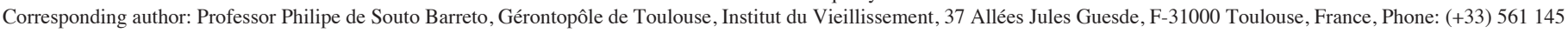
636, Fax: (+33) 561145 640, e-mail: philipebarreto81@yahoo.com.br

\begin{abstract}
Aging is the most important risk factor for the onset of several chronic diseases and functional decline. Understanding the interplays between biological aging and the biology of diseases and functional loss as well as integrating a function-centered approach to the care pathway of older adults are crucial steps towards the elaboration of preventive strategies (both pharmacological and non-pharmacological) against the onset and severity of burdensome chronic conditions during aging. In order to tackle these two crucial challenges, ie, how both the manipulation of biological aging and the implementation of a function-centered care pathway (the Integrated Care for Older People (ICOPE) model of the World Health Organization) may contribute to the trajectories of healthy aging, a new initiative on Gerosciences was built: the INSPIRE research program. The present article describes the scientific background on which the foundations of the INSPIRE program have been constructed and provides the general lines of this initiative that involves researchers from basic and translational science, clinical gerontology, geriatrics and primary care, and public health.
\end{abstract}

Key words: Gerosciences, biological aging, healthy aging, intrinsic capacity.

\section{Introduction}

Aging is a major risk factor for the development of several burdensome chronic diseases. From this ancient observation, a new research field has been built with the objective of understanding the interplay between aging and chronic diseases: Gerosciences (1-3). Indeed, the Gerosciences' area is born from the hypothesis that by manipulating biological aging processes, the onset of chronic diseases during aging might be prevented or postponed, and their severity decreased (4).

To date, the crucial role of aging on the onset of diseases and declines in functional outcomes has been observed mostly from an epidemiological perspective using a chronological approach, ie, chronological age. However, there is evidence supporting that aging (ie, biological and physiological processes) is a most reliable determinant of health than age (ie, the count of years lived) (5), being associated with health outcomes independently of individual's chronological age (6-8). Although the field of biological aging has made outstanding progresses in the past decades, evidence linking this knowledge with human healthy aging are still limited. Healthy aging is strongly determined by the maintenance of optimal levels of functional capacities, such as mobility, cognition, psychological, nutritional, and sensorial capacities; therefore, linking biological aging not only to chronic diseases, but also to the evolution of functional capacities $(9,10)$ is a research field that deserves to be explored.

Furthermore, the extent to which manipulating aging would modify health trajectories in the life course is almost completely unknown. In order to foster advances in the Gerosciences' area, a better understanding about how to measure biological aging (ie, determining the aging phenotype) $(11,12)$ as well as the definition of a set of robust and sensitive biomarkers (including traditional markers from biospecimens, but also digital and other markers) capable of predicting healthy/unhealthy aging as defined by the World Health Organization (WHO) (13) is an indispensable step. Such advances in the measurement of biological aging and definition of biomarkers would ultimately permit individuals' risk stratification early in the life-course. Moreover, such a set of biomarkers, allied to individuals' risk stratification, might inform timely pharmacological and non-pharmacological 


\section{THE JOURNAL OF FRAILTY \& AGING}

preventive strategies acting directly on aging, contributing to a healthy aging even in late ages.

In this context, the creation of a research platform with baseline and follow-up biological, clinical and digital data for both humans and animals of several chronological ages and functional capacity status constitutes a crucial element to propel discoveries in this broad domain of healthful aging. The objective of the present article is to describe the INSPIRE research program, an initiative aimed at fostering research in the field of Gerosciences and healthy aging.

\section{The intrinsic capacity framework: creating the bridge between Gerosciences and healthy aging}

Healthy aging was defined by the World Health Organization (WHO) as "the process of developing and maintaining the functional ability that enables well-being in older age" (13). In other words, healthy aging is not the absence of chronic diseases, but the ability to cope in daily life. Although the first foundations of the Gerosciences' field relate to the interplays between the biology of aging and the biology of diseases (1, 4), recent literature recognizes the importance of functional capacity and frailty for Gerosciences' investigations (14). Indeed, the Gerosciences' field investigates the fundamental mechanisms of biological aging; these fundamental mechanisms are shared by several organs and, once their deterioration exceeds the individual's resilience reserve, they will lead to clinical phenotypes (eg, frailty, disability, diseases, early mortality) (14) that may (or may not) be chronic diseases.

In recent years, experts from the field of biological aging converged into a list of nine major biological and physiological processes that determines aging, the so-called hallmarks of aging: genomic instability, telomere attrition, epigenetic alterations, loss of proteostasis, deregulated nutrient sensing, mitochondrial dysfunction, cellular senescence, stem cell exhaustion, and altered intercellular communication (15). Elements of the hallmarks of aging are associated to both declines on functional capacity and the onset and severity of chronic diseases. As examples, we can cite: a) low-grade chronic inflammation, a marker of altered intercellular communication, is associated with declines on cognitive (16), mobility (17) and psychological functions (16), but also with the onset and worsening severity of frailty and diseases (18, 19), such as cardiovascular and metabolic diseases; b) reduction in the repair capacity of muscle fibers, a marker of stem cells exhaustion, may lead to sarcopenia (20) (which is a disease) and decreases in mobility function.

Several other examples might be provided, but the important message to retain is that chronic diseases and body functions share similar biological and physiological processes that partly determine individual's health during aging. Therefore, it is plausible to accept that the Gerosciences field should embrace elements contributing to health $(9,21)$ other than chronic diseases, in particular the biological background affecting the levels of IC (body functions) and, thus, healthy aging.

\section{The INSPIRE research program: an overview}

INSPIRE is a research program dedicated to biological and healthy aging, aimed at constituting a bio-resource platform going from animals to humans, from cells to individuals, from research to clinical care. INSPIRE will provide clinical, biological and technological resources for research and development on aging open to both academic and business worlds in order to tackle one of the major societal challenges worldwide: promote healthy aging and prevent dependency.

INSPIRE is a public-private initiative that brings together internationally recognized experts from basic and translational science (in particular, in the fields of immunology, metabolism, and stroma), clinical gerontology (ie, researchers, but also physicians and nurses involved in clinical care), primary care and public health. Table 1 displays the main academic and care teams contributing to the INSPIRE initiative.

\section{INSPIRE main objectives}

1. Identifying biomarkers of aging. INSPIRE will provide clinical and digital data as well as biospecimens from both human and animals, to foster the identification and validation of (a set of) biomarkers of aging. Biomarkers are here understood in a broad meaning, going from traditional biomarkers from biofluids or other biomaterials and imaging, to alternative measurements, such as digital markers obtained from both innovative refined automated video and 3D photo analysis.

2. Implementing a function-centered healthcare pathway for aging. In the INSPIRE program, we will implement the Integrated Care for Older People (ICOPE) recommendations from the WHO; ICOPE recommends to focus the care of older individuals according to the five domains of intrinsic capacity (function-centered approach). INSPIRE will allow us to go beyond the simple implementation of ICOPE, by fostering investigations on both the biological changes related to healthy aging and the development of new technologies and metrics enabling the overtime monitoring of functions during aging.

\section{INSPIRE main milestones}

1. The creation of the INSPIRE Human Translational Cohort, with extensive biobanking. This human research cohort will foster discoveries of human markers (ie, biological, clinical, digital) of healthy aging capable of predicting functioning and resilience.

2. The creation of the INSPIRE Animal Cohort, with extensive biobanking. This animal cohort will be specifically dedicated to the discovery of biomarkers of aging, mechanisms of action, and target-specific investigations.

3. The implementation of the ICOPE program from the WHO in clinical practice, including the remote monitoring of 
THE INSPIRE RESEARCH INITIATIVE

Table 1

Academic and care teams contributing to the INSPIRE Program

University/research unity
Gerontopole of the Toulouse University Hospital
(CHU-Toulouse)

Inserm-Université Paul Sabatier UMR 1027 (Epidemiology and aging)

Inserm-Université Paul Sabatier, EFS, ENVT, UMR1031, ERL CNRS 5311 (StromaLab)

Inserm-Université Paul Sabatier UMR 1048 (Institute of Cardiovascular and Metabolic Diseases - I2MC)

Inserm-CNRS-Université Paul Sabatier UMR 1043 (Center for Pathophysiology of Toulouse Purpan CPTP)

Inserm-Université Paul Sabatier UMR 1214 (Toulouse Neuroimaging Center - ToNIC)

CNRS-Université Paul Sabatier UMR 5169 (Research Center on Animal Cognition - CRCA-CBI)

Digestive Health research Institute, Inserm U1220 (IRSD)

Main scientific expertise

Clinical research on geriatrics, with main focus on the full spectrum of the disablement cascade, including frailty, disability, and functional levels of mobility, cognition, and psychological capacity, as well as the role of lifestyle factors and interventions (eg, nutrition and exercise) of functioning-related declines.

Prevention of age-related conditions with a continuum between preventive clinical trials and translation from research findings into public health policy. Methodological expertise (Design and analyses of cohorts and large preventive trials).

Regenerative medicine based on mesenchymal stem/ stromal cells (MSC) and metabolism, focusing on adipose tissue as a physiological and therapeutic reservoir of mesenchymal and hematopoietic stem cells for repair processes.

Basic and translational research on metabolic (obesity, diabetes, dyslipidemia) and cardiovascular (atherosclerosis, renal and heart failure) diseases The research teams have a large expertise in hormone receptors, cell signaling, metabolic pathways, cell/ cell interactions and the mechanisms of tissue remodeling/regeneration. In the context of INSPIRE, the teams will investigate the interactions among biological aging, chronic metabolic/cardiovascular diseases and frailty.

Basic and translational research on the involvement of the innate and adaptive immune system in the pathophysiology of infectious and inflammatory diseases. In INSPIRE, the team ambitions to identify inflammatory biomarkers of aging and to provide therapeutic targets to prevent or revert ageassociated defects through the molecular analysis of immune cell dysfunction associated with aging.

Clinical and translational research programs on the main central nervous system diseases, using neurological, neuropsychological, neuropharmacological and neurosurgical investigations coupled with brain stimulation/ rehabilitation techniques and neuroimaging explorations, from single-photon emission computed tomography to positron emission tomography/ cyclotron and MRI systems.

Multidisciplinary and comparative study of cognitive processing in different animal models, from invertebrates to vertebrates. Basic and translationa research devoted to cognitive decline are developed with different approaches from mathematical models to behavioral phenotyping.

Basic research in the physiology and pharmacology of the digestive organs, including the liver, the intestine and the microbiota. Focus on iron metabolism and iron-related diseases (anemia or sarcopenia). Focus on microbiota analysis, hostmicrobiota interactions. Focus on visceral pain.

\section{Platforms/staff involved in INSPIRE}

Care: Regional Team for Dependency Prevention (primary care network with thousands of healthcare professionals); ambulatory services (eg, frailty day-hospital, memory clinics, consultations) Research: Clinical Research Center (about 30 people, partly involved in Inspire); Institute on Aging (about 16 people - fully dedicated to INSPIRE)

Research center composed of epidemiologists, physicians, pharmacists, primary care physicians, statisticians (near 60 subjects partly involved in INSPIRE)

-cell production process approved by the European Medicines Agency

-national infrastructure for in vivo and in vitro biosafety and quality control testing (about 50 people partly involved in INSPIRE)

Seven platforms (Bioinformatics, Cytometry, Transcriptomics, Cell Imaging, Lipidomics, Histology, Functional Proteomics) and the 14 research teams (about 280 people partly involved in INSPIRE) will participate in the INSPIRE project.

Research center composed of technical staff, scientists, professors, and physicians (about 200 individuals will be partly involved in INSPIRE)

- 3T MRI Philips scanner, upgraded for digital improvement, and the support staff (2 research engineers and technologists). Before collection of INSPIRE data, we will use available datasets (eg ADNI, MAPT) to set up and optimize a pipeline. line of processing and data analysis that will then be used on the INSPIRE data for the assessment of sarcopenia and brain function.

Research center composed of technical staff, scientists and professors (about 30 subjects will be partly involved in INSPIRE)

-The CBI Mouse Behavioral Core will be in charge of supervising the longitudinal phenotyping of mice in INSPIRE (index of frailty and cognitive decline)

Organoid Core Facility: Generates and stores cultures of mini-organs from tissue biopsies.

Research Teams: Potentially all the research teams of the Institute, but more specifically, one team on iron metabolism, one team on microbiota-host interactions and one team on visceral pain. 


\section{THE JOURNAL OF FRAILTY \& AGING}

IC domains. This implementation activity will give rise to the INSPIRE Clinical Care Cohort, which will provide information on the evolution of functioning in a real-life population and will inform about the feasibility (including in terms of using digital health tools) of implementing a function-centered model of care (instead of the traditional disease-centered model).

See Figure 1 for a schematic presentation of the INSPIRE program. We briefly describe below the most important characteristics of the three milestones of the INSPIRE Program.

\section{The INSPIRE Human Translational Cohort}

This is an observational study that will recruit about 1,000 subjects, men and women, aged 20 years-old or over (no upper limit for age), and affiliated to a social security scheme; will be excluded people having a severe disease with life expectancy lower than five years (lower than one year for the small fraction of INSPIRE participants who are disabled older adults), and those deprived of their liberty by administrative or judicial decision or under guardianship. The population of individuals 60 or over will be oversampled in order to capture major clinical events (eg, declines on functions, onset of disability, frailty, burdensome chronic diseases). Participants will be recruited mainly from the healthcare services (eg, ambulatory geriatrics services, medical records of primary care physicians located in different areas, with different social deprivation levels) in the city of Toulouse and surrounding regions, SouthWestern, France.
Participants will provide biospecimens: blood, urine, saliva, dental biofilm, nasopharyngeal/oropharyngeal swabbing, and skin swabbing and stripping. Several clinical measurements will be undertaken in all participants, including the five domains of IC (ie, locomotion, cognition, vitality/nutrition, psychological and sensorial capacities), frailty, functional ability, oral health, lifestyle (eg, physical activity (including objectively measured), diet, smoking, sun exposure), as well as participant-reported outcomes (eg, cognition, mobility, fatigue, social isolation). Subsamples of participants will have collected other biospecimens (eg, feces, hair bulb, skin biopsies) and/or will undertake more in-depth evaluations, such as Dual Energy X-ray Absorptiometry (DEXA), whole body and brain magnetic resonance imaging, cardiorespiratory fitness (maximum oxygen consumption $\left(\mathrm{VO}^{2} \mathrm{max}\right)$ and maximal aerobic power), and isokinetic muscle strength. Innovative digital assessments are also planned to be tested, such as home sensors (eg, for measuring walking speed and its variability in daily environment), automated video analysis of mobility, and 3D facial images for the detection of digital markers of aging.

Participants will be evaluated once a year for clinical assessments and for the collection of some biospecimens (eg, blood, saliva, urine). Between yearly waves of data collection, participants will record major clinical information, including adverse events (eg, new diagnosis, fracture), medical consultations, and changes in the prescribed drugs. Furthermore, participants will have their IC domains monitored (with or without the help of a caregiver) each 4-month through the use of either an app, developed in collaboration with WHO, a web platform, or through a phone call by a clinical/research

Figure 1

The INSPIRE program for Gerosciences and healthy aging

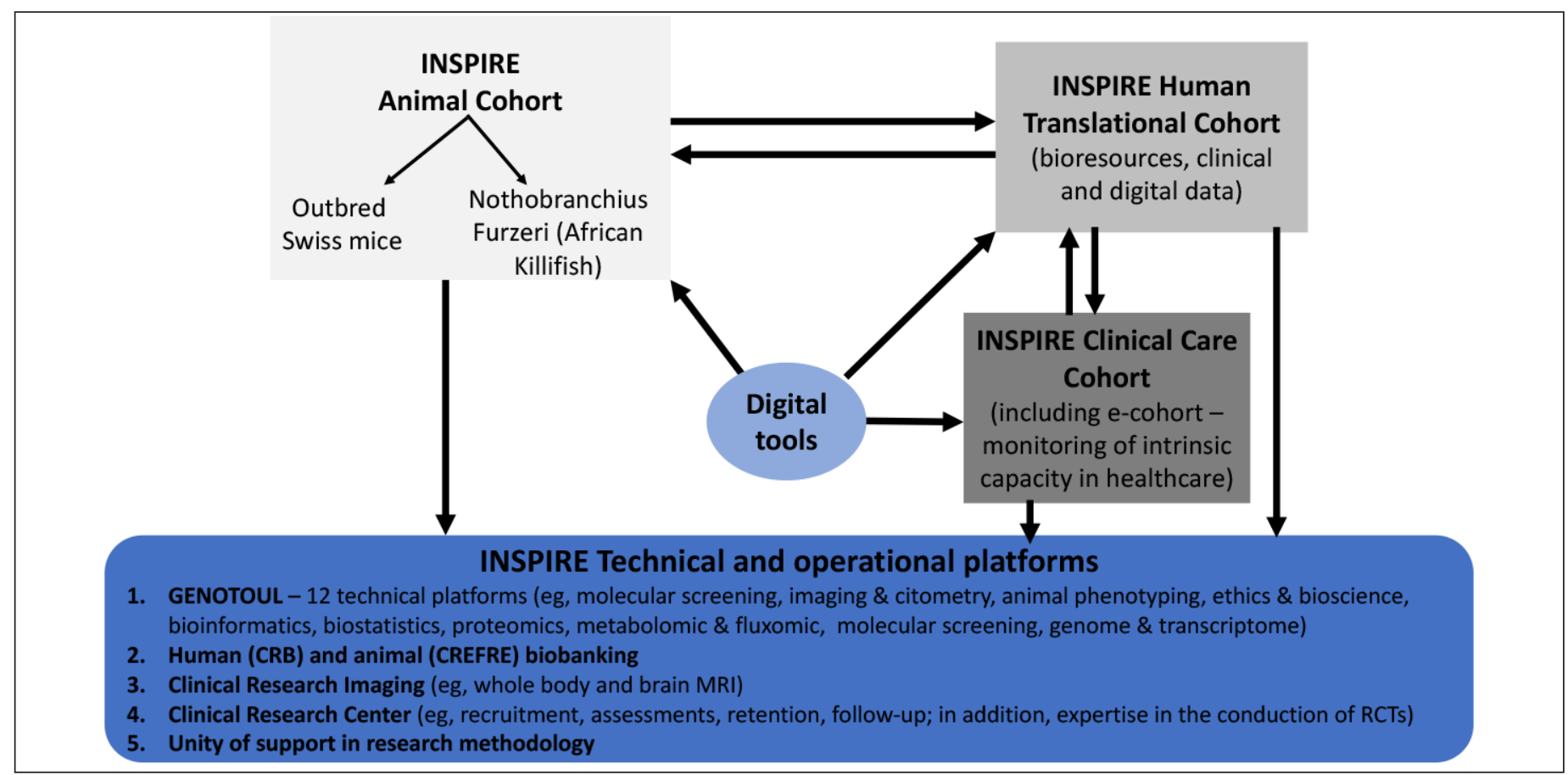


nurse. Once IC declines are confirmed, participants will have a thorough clinical assessment and blood sampling; such information will allow us to investigate the response of some biomarkers of aging at the time declines on IC are detected. All participants will receive the usual care on the basis of the recommendations from the WHO ICOPE model.

\section{The INSPIRE Animal Cohort}

The animal cohort has been generated to "mirror" the human translational cohort and facilitate the translation of results from basic research to humans and to the clinics. The main objective of the INSPIRE Animal Cohort is to define the relationship between the molecular mechanisms of cell premature senescence and frailty/accelerated aging. With this scope, we first attempted to design a mouse model as close as possible to human frailty/accelerated aging.

To mimic the genetic heterogeneity of human populations, we decided to use outbred Swiss mice, which have a "controlled" genetic heterogeneity and are currently used in longevity studies. Sedentarity and overweight approaches will be investigated because: i) in humans, they are known risk factors of frailty (22-25); ii) as compared to other experimental approaches, sedentarity and overweight are particularly suitable to promote metabolic and immune/inflammation dysfunctions largely described to be involved in progressive/long-term frailty; and iii) the INSPIRE investigators have international reputation in the field of metabolic diseases as well as immune/ inflammation dysfunctions.

The cohort will include between 1400 and 1500 (male and female) Swiss mice and four arms: control, high fat/high sucrose diet (HF/HSD), wheel (voluntary physical activity), $\mathrm{HF} / \mathrm{HSD}+$ wheel. Mice will be tested at the following ages: $6,12,18$ and 24 months corresponding to approximately 30 , 42, 56 and 69 years in humans. The number of mice has been defined based on i) male/female spontaneous and HF/HSDinduced mortality and ii) variability of experimental data. Mice will arrive at the central (CREFRE) animal facility when they will be 3-4 weeks old (specific pathogen free status) and they will be housed in a rate of four per cage. HF/HSD will be administrated from month 6 to month 24. Spontaneous physical activity will be continuously recorded and analyzed using cage electromagnetic plates connected to computers (Techniplast Connected Digital Ventilated Cages - DVC ${ }^{\mathrm{TM}}$ ). Voluntary physical activity will be determined by monitoring the access and use of wheels into the cages.

The animal cohort will be comprehensively phenotyped. This phenotyping will allow: i) firstly, to determine the onset of frailty in relation to HF/HSD and physical activity. Frailty will be defined at the different ages by a basic Frailty Index score including functional cognitive/motor (eg, Y-T-maze test, motor coordination test) and blood (eg, cell count and formula, inflammatory cytokines) tests. Additional tests will be performed to define an extended Frailty Index; ii) secondly, to investigate the role of different organ dysfunctions in the onset/progression of frailty and accelerated aging using "field-specific phenotyping" (eg, metabolic, cardiovascular, immuno/inflammatory, motor/cognitive, stem/progenitor cells) performed at the different ages; thirdly, to identify biomarkers' profiles predicting normal and accelerated aging using, as a first approach, omics "ID" for blood/urine (peptidome analysis) and feces (microbiota). The omics approaches will be then extended to tissues to identify novel tissue-specific putative biomarkers and therapeutic targets of frailty/accelerated aging.

INSPIRE will also use the accelerated aging model Nothobranchius Furzeri (African Killifish), the animal exhibiting one of the most reduced lifespan (4 to 6 months) among vertebrates; this model will be useful for isolating new hypothesis and validating identified mechanisms. Challenges, such as exercise, fasting or high-fat diet feeding, will be done in order to mimic the different conditions retrieved in human aging. Phenotyping of locomotion and cognition will be performed by camera video-tracking together with metabolic, immune and regenerative properties' assessments. All the experiments will be performed in males and females aged from 1 to 6 months. A fish-dedicated transgenesis platform will be built to facilitate the validation of the isolated targets.

After phenotyping, mice and fish will be sacrificed and blood, urine, feces and tissues will be collected for biobanking as appropriate.

\section{Implementation of the WHO ICOPE model: the INSPIRE Clinical Care Cohort}

The ICOPE model proposed by the WHO (26) is a functioncentered, instead of disease-centered, framework for the care management of people during aging, which focuses on the clinical domains of intrinsic capacity: locomotion, cognition, vitality/nutrition, psychological, and sensorial capacities. The ICOPE framework is structured into five steps, and the care pathway can be briefly described as follows: In ICOPE step1, IC clinical domains are screened; people with normal IC levels will receive general health advices (usual care), whereas those with low levels in at least one IC domain will go to ICOPE step-2 and will receive in-depth assessments; if the low IC levels are confirmed, their causes will be investigated; then, in step-3, a patient-centered personalized care plan will be established and monitored; referrals (ICOPE step-4) and caregiver and public health support (ICOPE step-5) may be part of the care plan. The WHO has recently launched the ICOPE app for tablet/smartphone (https://www.who.int/ageing/ health-systems/icope/en/), in which data (ie, step 1 and step 2 measurements) and information for the whole ICOPE care pathway can be recorded.

In collaboration with local (Regional Health Agency in the Region Occitanie) and national (French Ministry of Health) health authorities, the INSPIRE program will implement the ICOPE model in the clinical care. For this, primary care 


\section{THE JOURNAL OF FRAILTY \& AGING}

providers, in particular community nurses, in the Occitanie Region (South-Western, France), will use the ICOPE app for tablet/smartphone to implement the ICOPE pathway for each older adult they care. ICOPE step-1 (ie, screening for low IC levels) will be performed in all individuals; the other steps of the model may be performed according to available resources in the local care services/facilities. Moreover, a slightly modified version of the step-1 (with discrete/continuous IC variables) of the ICOPE app will be used for the remote monitoring of IC levels over time. All the data will be automatically transferred to a secured database and will be used to investigate the overtime evolution of IC domains.

Once discoveries (eg, biomarkers of aging) emerge from the INSPIRE human/animal cohorts and from other investigators around the world, some of them can be easily and quickly tested and validated in this real-life population composed of people followed by the healthcare professionals who implemented the ICOPE care pathway.

\section{What are the novelties brought about by the INSPIRE research program?}

One of the hallmarks of INSPIRE relates to the fact this program will cover longitudinally a large age range, from young to very old individuals, and the whole spectrum of the disablement cascade, from robustness (healthy people) to frail to disabled individuals. Moreover, by running two research cohorts in parallel, one with humans the other with animals, we expect to facilitate the cross-talks between human research and experimental models, what will probably speed up the discovery process. It is noteworthy that the animal cohort will mimic this diversity in functional status by providing both healthy and frail animal models to investigations.

Furthermore, in the INSPIRE Human Translational Cohort, clinical assessments and biological material (eg, blood) will be obtained at several time-points and immediately after IC declines are detected; to the best of our knowledge no study has collected biospecimens at the moment clinical symptomatology related to loss of function appears. Indeed, several past and/or on-going studies on aging and biomarkers, including very large studies, such as the UK biobank(27), the All of Us research program (https://allofus.nih.gov/) or still the Canadian Longitudinal Study on Aging(28) (http:// www.clsa-elcv.ca/), have collected blood, urine, and/or saliva. However, biospecimens' collection was done only at baseline or at regular pre-defined time intervals, such as every three years. The issue with these procedures is that clinically meaningful declines in IC as well as the onset of other clinical conditions may happen between two consecutive data collection visits and the biological changes determining the eruption of phenotypic manifestations may, then, be lost because the clinical condition may have been treated before the next wave of data collection. Moreover, the regular remote monitoring of IC will allow us to investigate the overtime fluctuations in IC domains more closely as well as to find out the predictors (eg, biological, behavioral, environmental, and others) of resilience during aging.

Another major innovative aspect of INSPIRE relates to digital markers of aging, which have been only modestly investigated in previous studies. In the INSPIRE human cohort, we are planning to equip initially 30 homes (projections are to equip up to 100 homes) with original sensors that collect daily life data in a continuous flow; this will allow us to investigate their associations with both biological markers of aging and well-established clinical assessments. Another innovative digital approach we are planning to undertake is automated video analysis of mobility as well as 3D facial images; by employing big data techniques, we expect to be able to detect digital markers of mobility decline and biological aging, respectively.

Finally, by implementing the ICOPE model in routine care, the INSPIRE program will be contributing to the change in the care approach to aging, replacing the current disease-centered model by a function-centered healthcare pathway. Furthermore, by following the patients overtime through the ICOPE app, this real-life population may constitute a pool of potential participants for the activities of the INSPIRE program (eg, feasibility of the remote monitoring of IC levels, validation of biomarkers, etc). See Box 1 for a summary of the main novelties of the INSPIRE program.

\section{Box 1}

New aspects of the INSPIRE program

\begin{abstract}
Although there are Institutions (eg, the Buck Institute ; https:// www.buckinstitute.org/) dedicated to investigate biological aging and projects devoted to study biomarkers of aging, such as the European Mark-Age Consortium(29), although animal cohorts dedicated to investigations on biological aging (eg, National Institute on Aging rodent cohort : https://www.nia.nih.gov/research/dab/ aged-rodent-colonies-handbook) and Gerosciences(30) exist, and although currently available longitudinal human studies with several years of follow-up may be used to examine the biomarkers of aging and provide metrics of human biological age(31), to the best of our knowledge the INSPIRE program is one of the first research initiatives on Gerosciences to:
\end{abstract}

1. Provide a comprehensive approach to Gerosciences and healthy aging, going from basic science to clinical research, and then to the healthcare system. For this, INSPIRE includes animal models (mice and fish), human bioresources and clinical and digital outcomes, and the implementation of integrated care for older people (ICOPE), a function- and person-centred approach, in the healthcare system.

2. Collect both clinical and biological data at the moment declines on intrinsic capacity are detected and confirmed, which will contribute to improving the understanding about molecular and physiological mechanisms involved in healthy aging 


\section{THE INSPIRE RESEARCH INITIATIVE}

\section{Discussion}

To the best of our knowledge, the INSPIRE program is one of the major international research and care initiatives on Gerosciences and healthy aging. INSPIRE has the potential to strongly contribute to the discovery of biomarkers and mechanisms of aging, paving the way for the development of pharmacological and non-pharmacological approaches targeting biological aging. Moreover, by associating the healthcare pathway of older adults to research (32), INSPIRE will inform about both the feasibility of evaluating IC levels in primary care services and the remote overtime monitoring of IC in this population; investigations of the associations between the ICOPE healthcare pathway and clinical outcomes $(33,34)$ (in particular, the prevention of disability and dependency) and health economics may also be explored.

The expected impact of the INSPIRE program relates to both research and care on aging and health. First, due to its innovative procedures, characterized by clinical and biological data collection at the moment IC declines are detected and confirmed, this project will contribute to improving our understanding about molecular and physiological mechanisms involved in healthy aging; such mechanisms may interact or not with disease-specific biological changes. Ultimately, INSPIRE may contribute to establish a set of biomarkers of healthy aging. Second, by following and monitoring the IC levels of participants over time, this research and care program will provide invaluable information about a new, function-centered healthcare pathway, which would be in agreement with WHO recommendations for an integrated care for older people (26). Third, the digital part of the study may lead to the identification of markers of healthy/unhealthy aging; when validated, such measures have the major advantage of being non-invasive and easily assessable, which means they have the potential to be translated into practice.

In sum, aging is complex and is a major risk factor for most chronic diseases and dependency in late life; we believe its underpinning biological processes can be manipulated and, then, contribute to healthy aging $(35,36)$. The exponential growth of the aging population, the potential explosion of disabling conditions in the coming decades and the associated catastrophic increase in healthcare costs, ask for urgent solutions. The INSPIRE program will give rise to a unique center gathering expertise from basic and translational science, clinical research, and geriatric care, with the ultimate goal of preventing adverse health consequences of aging, delaying their onset or reducing their severity. The challenges for the INSPIRE program are multiple and reflect challenges faced by the fields of Gerosciences and healthy aging, including: the coordination among researchers from different research areas, from basic science, translational, and clinical research to public health and clinical care; contributing to changing the disease-based healthcare culture, which has been established for decades; identifying biomarkers of aging that are (or that can be in the near future) easily assessable and cheap for facilitating its use in routine clinical care. Although these and other challenges cannot be overcome in a short time interval, we consider that applying the principles of Gerosciences to foster discoveries that can contribute to healthy aging and that can be translated into clinical practice is an exciting and worthy initiative.

Acknowledgments: The Inspire Platform was supported by grants from the Region Occitanie/Pyrénées-Méditerranée (Reference number: 1901175), the European Regional Development Fund (ERDF) (Project number: MP0022856), and the Inspire Chairs of Excellence funded by: Alzheimer Prevention in Occitania and Catalonia (APOC), EDENIS, KORIAN, Pfizer, Pierre-Fabre.

Conflcit of interest: All authors of the paper "The INSPIRE research initiative: a program for GeroScience and healthy aging research going from animal models to humans and the healthcare system" declare no Conflicts of Interest related to this manuscript.

Open Access: This article is distributed under the terms of the Creative Commons Attribution 4.0 International License (http://creativecommons.org/licenses/by/4.0/), which permits use, duplication, adaptation, distribution and reproduction in any medium or format, as long as you give appropriate credit to the original author(s) and the source, provide a link to the Creative Commons license and indicate if changes were made.

\section{References}

1. Sierra F, Kohanski R. Geroscience and the trans-NIH Geroscience Interest Group, GSIG. GeroScience. 2017;39(1):1-5.

2. Sierra F. The Emergence of Geroscience as an Interdisciplinary Approach to the Enhancement of Health Span and Life Span. Cold Spring Harb Perspect Med. 2016 Apr 1;6(4):a025163.

3. Sierra F. Moving Geroscience Into Uncharted Waters. J Gerontol A Biol Sci Med Sci. 2016;71(11):1385-7.

4. Kennedy BK, Berger SL, Brunet A, Campisi J, Cuervo AM, Epel ES, et al Geroscience: linking aging to chronic disease. Cell. 2014 Nov 6;159(4):709-13.

5. Levine ME. Modeling the rate of senescence: can estimated biological age predict mortality more accurately than chronological age? J Gerontol A Biol Sci Med Sci. 2013 Jun;68(6):667-74.

6. Belsky DW, Moffitt TE, Cohen AA, Corcoran DL, Levine ME, Prinz JA, et al. Eleven Telomere, Epigenetic Clock, and Biomarker-Composite Quantifications of Biological Aging: Do They Measure the Same Thing? Am J Epidemiol. 2018 01;187(6):1220-30.

7. Hastings WJ, Shalev I, Belsky DW. Comparability of biological aging measures in the National Health and Nutrition Examination Study, 1999-2002. Psychoneuroendocrinology. 2019 Aug;106:171-8.

8. Belsky DW, Caspi A, Houts R, Cohen HJ, Corcoran DL, Danese A, et al. Quantification of biological aging in young adults. Proc Natl Acad Sci U S A. 2015 Jul 28;112(30):E4104-4110.

9. Seals DR, Justice JN, LaRocca TJ. Physiological geroscience: targeting function to increase healthspan and achieve optimal longevity. J Physiol. $2016 \mathrm{Apr}$ 15;594(8):2001-24.

10. Seals DR, Melov S. Translational geroscience: emphasizing function to achieve optimal longevity. Aging. 2014 Sep;6(9):718-30.

11. Ferrucci L, Levine ME, Kuo P-L, Simonsick EM. Time and the Metrics of Aging. Circ Res. 2018 14;123(7):740-4.

12. Margolick JB, Ferrucci L. Accelerating aging research: how can we measure the rate of biologic aging? Exp Gerontol. 2015 Apr;64:78-80.

13. World Health Organization. World report on ageing and health. [Internet]. WHO Press; 2015. Available from: https://www.who.int/ageing/events/world-report-2015launch/en/

14. Ferrucci L, Gonzalez-Freire M, Fabbri E, Simonsick E, Tanaka T, Moore Z, et al Measuring biological aging in humans: A quest. Aging Cell. 2019 Dec 12;e13080.

15. López-Otín C, Blasco MA, Partridge L, Serrano M, Kroemer G. The hallmarks of aging. Cell. 2013 Jun 6;153(6):1194-217.

16. Giudici KV, de Souto Barreto P, Guerville F, Beard J, Araujo de Carvalho I, Andrieu $\mathrm{S}$, et al. Associations of $\mathrm{C}$-reactive protein and homocysteine concentrations with the 


\section{THE JOURNAL OF FRAILTY \& AGING}

impairment of intrinsic capacity domains over a 5-year follow-up among communitydwelling older adults at risk of cognitive decline (MAPT Study). Exp Gerontol. 2019 Nov; 127:110716.

17. Penninx BWJH, Kritchevsky SB, Newman AB, Nicklas BJ, Simonsick EM, Rubin $\mathrm{S}$, et al. Inflammatory markers and incident mobility limitation in the elderly. $\mathrm{J}$ Am Geriatr Soc. 2004 Jul;52(7):1105-13.

18. Marzetti E, Picca A, Marini F, Biancolillo A, Coelho-Junior HJ, Gervasoni J, et al. Inflammatory signatures in older persons with physical frailty and sarcopenia: The frailty 'cytokinome' at its core. Exp Gerontol. 2019 Jul 15;122:129-38.

19. Fulop T, Witkowski JM, Olivieri F, Larbi A. The integration of inflammaging in agerelated diseases. Semin Immunol. 2018;40:17-35.

20. Blau HM, Cosgrove BD, Ho ATV. The central role of muscle stem cells in regenerative failure with aging. Nat Med. 2015 Aug;21(8):854-62.

21. Kaeberlein M. How healthy is the healthspan concept? GeroScience. 2018 Aug 6;40(4):361-4.

22. García-Esquinas E, Andrade E, Martínez-Gómez D, Caballero FF, López-García E, Rodríguez-Artalejo F. Television viewing time as a risk factor for frailty and functional limitations in older adults: results from 2 European prospective cohorts. Int J Behav Nutr Phys Act. 2017 26;14(1):54

23. Crow RS, Lohman MC, Titus AJ, Cook SB, Bruce ML, Mackenzie TA, et al. Association of Obesity and Frailty in Older Adults: NHANES 1999-2004. J Nut Health Aging. 2019;23(2):138-44.

24. Herr M, Cesari M, Landre B, Ankri J, Vellas B, Andrieu S, et al. Factors associated with changes of the frailty status after age 70: Findings in the MAPT study. Ann Epidemiol. 2019 Jun;34:65-70.e1.

25. Ho H-E, Yeh C-J, Chu W-M, Lee M-C. Midlife Body Mass Index Trajectory and Risk of Frailty 8 Years Later in Taiwan. J Nutr Health Aging. 2019;23(9):849-55.

26. WHO । WHO Guidelines on Integrated Care for Older People (ICOPE) [Internet] WHO. [cited 2019 Jan 25]. Available from: http://www.who.int/ageing/publications/ guidelines-icope/en/

27. Sudlow C, Gallacher J, Allen N, Beral V, Burton P, Danesh J, et al. UK biobank: an open access resource for identifying the causes of a wide range of complex diseases of middle and old age. PLoS Med. 2015 Mar;12(3):e1001779.
28. Raina PS, Wolfson C, Kirkland SA, Griffith LE, Oremus M, Patterson C, et al The Canadian longitudinal study on aging (CLSA). Can J Aging Rev Can Vieil. 2009 Sep;28(3):221-9.

29. Bürkle A, Moreno-Villanueva M, Bernhard J, Blasco M, Zondag G, Hoeijmakers JHJ, et al. MARK-AGE biomarkers of ageing. Mech Ageing Dev. 2015 Nov;151:2-12.

30. Kaeberlein M, Creevy KE, Promislow DEL. The dog aging project: translational geroscience in companion animals. Mamm Genome Off J Int Mamm Genome Soc. 2016;27(7-8):279-88.

31. Kuo P-L, Schrack JA, Shardell MD, Levine M, Moore AZ, An Y, et al. A roadmap to build a phenotypic metric of ageing: insights from the Baltimore Longitudinal Study of Aging. J Intern Med. 2020 Feb 27;

32. Takeda C., Guyonnet S., Sumi Y. et al.. Integrated Care for Older People and the Implementation in the INSPIRE Study. J Prev Alz Dis 2020;2(7):70-74

33. Vellas B, Scrase D, Rosenberg GA, Andrieu S, Araujo de Carvalho I, Middleton LT. Editorial: WHO Guidelines on Community-Level Interventions to Manage Declines in Intrinsic Capacity: The Road for Preventing Cognitive Declines in Older Age? J Prev Alzheimers Dis. 2018;5(3):165-167. doi: 10.14283/jpad.2018.26. PubMed PMID: 29972207.

34. Beard, J.R. , Linking Geroscience and Integrated Care to Reinforce Prevention, J Prev Alz Dis 2020;2(7):68-69

35. Guerville F, De Souto Barreto P, Ader I, Andrieu S, Casteilla L, Dray C, Fazilleau N, Guyonnet S, Langin D, Liblau R, Parini A, Valet P, Vergnolle N, Rolland Y, Vellas B. Revisiting the Hallmarks of Aging to Identify Markers of Biological Age. J Prev Alzheimers Dis. 2020;7(1):56-64. doi: 10.14283/jpad.2019.50. PubMed PMID: 32010927

36. Sierra F. Editorial: Geroscience and the Role of Aging in the Etiology an Management of Alzheimer's Disease. J Prev Alzheimers Dis. 2020;7(1):2-3. doi: 10.14283/jpad.2019.49. PubMed PMID: 32010917. 